Pacific Journal of Mathematics

INTEGRAL CLOSURE OF DIFFERENTIAL RINGS 


\section{INTEGRAL CLOSURE OF DIFFERENTIAL RINGS}

\section{EDWARd C. Posner}

We prove that a commutative differentiably simple ring of characteristic zero finitely generated over its field of constants is integrally closed in its field of quotients. (A ring is differentiably simple if it has non-trivial multiplication and has no ideal invariant under a given family of derivations; i.e., has no differential ideals other than (0). The field of constants is the subring of the ring annihilated by each derivation of the family of derivations.) The result of the first sentence is used to obtain a condition that the powers of an element of a function field in one variable form an integral basis. The following results from [1] will be used: A commutative differentiably simple ring of characteristic zero is an integral domain whose ring of constants is a field. Crucial is the following lemma:

Lemma. Let $F$ be a field of characteristic zero; $x_{1}, \cdots, x_{n}$ be $n$ independent transcendentals over $F ; y_{1}, \cdots, y_{q}$ be integral over $x_{1}, \cdots$, $x_{n}$; and $d$ an $F$-derivation of $F[x, y]$ into itself. Then $d$ (or rather its natural extension to $F(x, y)$ ) sends $O_{x}$ (the set of elements of $F(x, y)$ integral over $x_{1}, \cdots, x_{n}$ ) into itself.

Proof. In general any $F$-derivation of $F(x, y)$ into itself can be written as

$$
d=\sum_{i=1}^{n} A_{1} \frac{\partial}{\partial x_{i}},
$$

$A_{i}$ elements of $F(x, y), 1 \leqq i \leqq n$, Further, $d$ maps $F[x, y]$ into itself if and only if $d\left(x_{i}\right)$ is in $F[x, y]$ for each $i$ and $d\left(y_{j}\right)$ is in $F[x, y]$ for each $j$. The first set of conditions is equivalent to the condition that $A_{i}$ be in $F[x, y]$ for each $i$.

In order to be able to use power series, we assume that $F$ is algebraically closed. For if not, let $\bar{F}$ be its algebraic closure. Let $d$ also be the extension of $d$ to $\bar{F}(x, y)$. Since $d$ sends $\bar{F}[x, y]$ into itself, $d$ send $\bar{O}_{x}$ into itself, where $\bar{O}_{x}$ denotes the ring of integral functions of $\bar{F}(x, y)$. A fortiori, $d$ sends $O_{x}$ into $\bar{O}_{x}$. But $\bar{O}_{x} \cap F[x, y]=O_{x}$ so actually $d$ sends $O_{x}$ into itself as required.

Let $P$ be a place of $F(x, y)$ over $F$ which has residue field $F$ and which is finnite on $x_{1}, \cdots, x_{n}$. We will prove that if $g$, in $F(x, y)$, is finite at $P, d(g)$ is finite at $P$. Let $a_{i}$ denote the residue of $x_{i}$ at $P$; then there exist uniformizing parameters $t_{1}, \cdots, t_{n}$ at $P$ such that

Received August 13, 1959, and in revised form January 23, 1960. 
$x_{i}-a_{i}$ is a positive integral power of $t_{i}$, say $x_{i}-a_{i}=t_{i}^{p_{i}}$. Every element $B$ of $F(x, y)$ finite at $P$ has a power series in $t_{1}, \cdots, t_{n}$ with coefficients in $F$. We call the smallest power of $t_{i}$ occurring in this series the $i$-order of $B$ at $P$, and denote it by $\operatorname{ord}_{P, i} B$; the definition of $\operatorname{ord}_{P, i} B$ extends to arbitrary elements $B$ of $F(x, y)$ in an obvious way. Fixing $i$, we see that if $\operatorname{ord}_{P, i} d(B) \geqq \operatorname{ord}_{P, i} B$ for every $B$ finite at $P$ then $\operatorname{ord}_{P, i} d(B) \geqq 0$ for every such $B$. Suppose there exists some $B$ finite at $P$ with $\operatorname{ord}_{P, i} d(B)<\operatorname{ord}_{P, i} B$. Then $\alpha_{i}-p_{i}<0$, where $\alpha_{i}=\operatorname{ord}_{P, i} A_{i}$, so that $r_{i}=p_{i}-\alpha_{i}>0$, and $\operatorname{ord}_{P, i} B=r_{i}+\operatorname{ord}_{P, i} d B$ for every $(B)$ in $F(x, y)$ with $\operatorname{ord}_{P, i} B \neq 0$. Since $d$ maps $F[x, y]$ into itself, the only values which ord $\operatorname{or}_{P, i} B$ can have when $B$ is in $F[x, y]$ are integral multiples of $r_{i}$, for otherwise some element of $F[x, y]$ would have negative $i$-order. Since $t_{1}, \cdots, t_{n}$ are uniformizing parameters, it follows that $r_{i}=1$, for otherwise we could replace $t_{i}$ by $t_{i}^{r_{i}}$. Thus, $d$ drops positive $i$-orders by 1 , so that $\operatorname{ord}_{P, i} d(B) \geqq 0$ for every $B$ finite at $P$. Since this holds for every $i, d(B)$ is finite at $P$ whenever $B$ is. Since this holds for every $P$, we conclude that $d$ maps $O_{x}$ into itself.

TheOREM 1. Let $F$ be a field of characteristic zero, $A=F\left[z_{1}, z_{2}, \cdots, z_{k}\right]$ a commutative finitely generated ring extension of $F$. Let $D$ be a (finite or infinite) family of derivations of $A$ into itself over $F$. Let $A$ be differentiably simple under $D$. Then $A$ is integrally closed in its quotient field $K$.

Proof. $A$ is an integral domain by (1). By Noether's Normalization Lemma, we can write $A=F\left[x_{1}, \cdots, x_{n} ; y_{1}, \cdots, y_{q}\right]$, with $n$ the transcendence degree of $K / F$ and $y_{1}, \cdots, y_{q}$ interal over $x_{1}, \cdots, x_{n}$. To prove $A=O_{x}$, let $I$ denote the conductor of $O_{x}$, that is, the set of elements $u$ of $F[x, y]$ such that $u \cdot O_{x} \subset F[x, y]$; by [3], pp. 271-2, prop. 6, $I$ is a non-zero ideal of $F[x, y]$. To prove $I$ differential under $D$, let $d$ be in $D, h$ be in $I, g$ be in $O_{x}$. Then $h \cdot g$ is in $F[x, y], d(h \cdot g)$ is in $F[x, y], d(h) g+h d(g)$ is in $F[x, y]$. Now $d(g)$ is in $O_{x}$ by the lemma so $h d(g)$ is in $F[x, y]$ since $h$ is in $I$. Then $d(h) g$ is in $F[x, y], I$ is differential under $D$. Then $I=F[x, y]$ so $1 \cdot O_{x} \subset F[x, y], O_{x}=F[x, y]$ as promised.

REMARK. $D$ can always be taken to be finite since the derivations of $F[x, y]$ into itself form a finite $F[x, y]$-module.

The converse of Theorem 1 is false, i.e., there are integrally closed finitely generated domains which are not differentiably simple under any family of $F$-derivations. For example, let $y^{2}=x_{1}^{3}+x_{2}^{3}$. Then $F[x, y]=O_{x}$ but is not differentiably simple over $F$. In fact, the ideal $\left(x_{1}, x_{2}, y\right)$ of $F[x, y]$ is differential for any derivation, as is easy to see. But when $n=1$, we do have the converse. (For background material, see [2], 
pp. 83-88.)

THEOREM 2. Let $K$ be a function field in one variable over a field $F$ of characteristic zero, and let $x$ be an element of $K$ transcendental over $F$. Let $O_{x}$ denote the set of elements of $K$ integral over $x$. Then $O_{x}$ is differentiably simple with field of constants $F$ under a family of two or fewer derivations.

Proof. First we shall specify the derivations. $O_{x}$ is a Dedekind ring, i.e., every ideal of $O_{x}$ is invertible. Let $K=F(x, y)$ with $y$ integral over $x$ and let $f(x, y)=0$ be the irreducible monic for $y$. Define $d$ on $K$ by

$$
d(g(x, y))=\frac{\partial g}{\partial x} \frac{\partial f}{\partial y}-\frac{\partial g}{\partial y} \frac{\partial f}{\partial x} .
$$

This is well-defined, and $d$ sends $O_{x}$ into itself by the lemma. Let $J$ be the ideal of $O_{x}$ generated by the values of $d$ of integral elements. $J$ is invertible, so there exist $h_{i}(x, y)$ in $K, 1 \leqq i \leqq q$, such that $h_{i} d$ sends $O_{x}$ into itself and such that there exist $u_{i}$ in $O_{x}, 1 \leqq i \leqq q$, with $\sum_{i=1}^{q} h_{i} d\left(u_{i}\right)=1$. ( $q$ can be taken to be 2 . For $J$ is generated by $f_{x}$ and $f_{y}$, since $d(M(x, y))=f_{y} M_{x}-f_{x} M_{y}$ for $M$ in $K . q$ can be taken to be 1 if and only if $J$ is principal, which need not occur.) The family $D$ is $\left\{h_{1} d, \cdots, h_{q} d\right\}$. To prove $O_{x}$ differentiably simple under $D$, suppose the contrary. As in the preceding and following theorems, $F$ may be assumed to be algebraically closed. If $O_{x}$ has a non-zero differential ideal, it has a maximal differential ideal $I$, since $O_{x}$ has a unit. $O_{x}^{2}$ is not contained in $I$, so by Theorem 4 of [1], $I$ is prime. But every prime ideal of $O_{x}$ is maximal; in fact, if $w$ belongs to $O_{x}$, there is a $\lambda$ in $F$ with $w-\lambda$ in $I$. Since $I$ is differential for $D, h_{i} d(w)-h_{i} d(\lambda)$ is in $I$, $1 \leqq i \leqq q, h_{i} d(w)$ is in $I, 1 \leqq i \leqq q$. That is, $h_{i} d(w)$ is in $I$ for all $w$ in $O_{x}$. Then $\sum_{i=1}^{q} h_{i} d\left(u_{i}\right)=1,1$ is in $I, I=O_{x}$. This contradiction proves that $O_{x}$ has no differential ideals. Its field of constants is $F$. For if $u$ is in $F(x, y)$ and $d(u)=0$ then $(d / d x)(u)=0$, so that $u$ belongs to $F$.

TheOREm 3. Let $K, F, x, O_{x}$ be as in the hypothesis of Theorem 2. Let $R$ be an order of $O_{x}$ and let $y$ be an element of $K$ integral over $x$ with irreducible monic $f$ such that $K=F(x, y)$. Then $R=O_{x}$ if and only if $y$ belongs to $R$ and the ideal $J$ in $R$ generated by $f_{x}$ and $f_{y}$ is invertible.

Proof. If $R=O_{x}$, then $y$ belongs to $R$ and every ideal in $R$ is invertible. Conversely, suppose that $y$ belongs to $R$ and that $J$, the ideal generated in $R$ by the values of $d$, is invertible. (Here $d$ is the same derivation as in Theorem 2.) That is, assume that there exist $h_{i}$ 
in $K, 1 \leqq i \leqq q$, with $h_{i} d$ sending $R$ into itself, and elements $v_{i}$ in $R$, $1 \leqq i \leqq q$, with $1=\sum_{i=1}^{q} h_{i} d\left(v_{i}\right)$. We shall prove $R$ differentiably simple under $D=\left\{h_{i} d, \cdots, h_{q} d\right\}$. It is known that every prime ideal of $R$ is maximal; it fact, if $I$ is a prime ideal of $R$, and $w$ is an element of $R$, there is a $\lambda$ in $F$ with $w-\lambda$ in $I$. If $R$ has a differential ideal, it has a maximal differential ideal, and one proceeds as in Theorem 2 . So $R$ is differentiably simple under $D$. By Theorem $1, R$ is integrally closed in $K$, i.e., $R=O_{x}$ as required.

As an illustration, let $K=F(x, y)$ with $f(x, y)=y^{n}-P(x)=0$, $n \geqq 1, P$ a polynomial in $x$ with no repeated roots. Here $R=F[x, y]$. Let us examine the ideal in $F[x, y]$ generated by $f_{x}$ and $f_{y}$, i.e., by $P^{\prime}(x)$ and $y^{n-1}$. This ideal contains $y^{n-1} y=y^{n}=P(x)$ and $p^{\prime}(x) . \quad P(x)$ and $P^{\prime}(x)$ have no common factor, so there are polynomials $Q(x)$ and $S(x)$ with $Q P+S P^{\prime}=1$. Then the ideal generated by $f_{x}$ and $f_{y}$ is $F[x, y]$ and so is trivially invertible. We conclude $F[x, y]=O_{x}$.

\section{BIBLIOGRAPHY}

1. E. C. Posner, Differentiably simple rings, Proc. Amer. Math. Soc., 11 (1960), 337343.

2. B. L. Van der Waerden, Modern algebra. Volume II. New York: Frederick Ungar Publishing Company, 1950.

3. A. Weil, Foundations of algebraic geometry, New York: American Mathematical Society, 1946.

THE UNIVERSITY OF WISCONSIN

MADISON, WISCONSIN 


\section{PACIFIC JOURNAL OF MATHEMATICS}

\section{EDITORS}

David GILbarg

Stanford University

Stanford, California

\section{F. H. BRowneLL}

University of Washington

Seattle 5, Washington

\section{A. L. Whiteman}

University of Southern California Los Angeles 7, California

\section{J. PAIGe}

University of California

Los Angeles 24, California

\section{ASSOCIATE EDITORS}
E. F. BECKENBACH
T. M. CHERRY
D. DERRY

\author{
E. HEWITT \\ A. HORN \\ L. NACHBIN
}

\author{
M. OHTSUKA \\ H. L. ROYDEN \\ M. M. SCHIFFER
}

E. SPANIER

E. G. STRAUS

F. WOLF

\section{SUPPORTING INSTITUTIONS}

\author{
UNIVERSITY OF BRITISH COLUMBIA \\ CALIFORNIA INSTITUTE OF TECHNOLOGY \\ UNIVERSITY OF CALIFORNIA \\ MONTANA STATE UNIVERSITY \\ UNIVERSITY OF NEVADA \\ NEW MEXICO STATE UNIVERSITY \\ OREGON STATE COLLEGE \\ UNIVERSITY OF OREGON \\ OSAKA UNIVERSITY \\ UNIVERSITY OF SOUTHERN CALIFORNIA
}

\author{
STANFORD UNIVERSITY \\ UNIVERSITY OF TOKYO \\ UNIVERSITY OF UTAH \\ WASHINGTON STATE COLLEGE \\ UNIVERSITY OF WASHINGTON \\ AMERICAN MATHEMATICAL SOCIETY \\ CALIFORNIA RESEARCH CORPORATION \\ HUGHES AIRCRAFT COMPANY \\ SPACE TECHNOLOGY LABORATORIES \\ NAVAL ORDNANCE TEST STATION
}

\footnotetext{
Mathematical papers intended for publication in the Pacific Journal of Mathematics should be typewritten (double spaced), and the author should keep a complete copy. Manuscripts may be sent to any one of the four editors. All other communications to the editors should be addressed to the managing editor, L. J. Paige at the University of California, Los Angeles 24, California.

50 reprints per author of each article are furnished free of charge; additional copies may be obtained at cost in multiples of 50 .
}

The Pacific Journal of Mathematics is published quarterly, in March, June, September, and December. The price per volume (4 numbers) is $\$ 12.00$; single issues, $\$ 3.50$. Back numbers are available. Special price to individual faculty members of supporting institutions and to individual members of the American Mathematical Society: $\$ 4.00$ per volume; single issues, $\$ 1.25$.

Subscriptions, orders for back numbers, and changes of address should be sent to Pacific Journal of Mathematics, 2120 Oxford Street, Berkeley 4, California.

Printed at Kokusai Bunken Insatsusha (International Academic Printing Co., Ltd.), No. 6, 2-chome, Fujimi-cho, Chiyoda-ku, Tokyo, Japan.

PUBLISHED BY PACIFIC JOURNAL OF MATHEMATICS, A NON-PROFIT CORPORATION

The Supporting Institutions listed above contribute to the cost of publication of this Journal, but they are not owners or publishers and have no responsibility for its content or policies. 


\section{Pacific Journal of Mathematics}

\section{Vol. 10, No. $4 \quad$ December, 1960}

M. Altman, An optimum cubically convergent iterative method of inverting a linear bounded operator in Hilbert space . . . . . . . . . . . . . . . . . . . . . . . . . . 1107

Nesmith Cornett Ankeny, Criterion for rth power residuacity ................. 1115

Julius Rubin Blum and David Lee Hanson, On invariant probability measures I . . . . . 1125

Frank Featherstone Bonsall, Positive operators compact in an auxiliary topology ..... 1131

Billy Joe Boyer, Summability of derived conjugate series . . . . . . . . . . . . . . . . 1139

Delmar L. Boyer, A note on a problem of Fuchs . . . . . . . . . . . . . . . . . 1147

Hans-Joachim Bremermann, The envelopes of holomorphy of tube domains in infinite

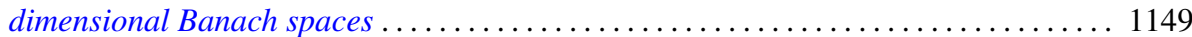

Andrew Michael Bruckner, Minimal superadditive extensions of superadditive

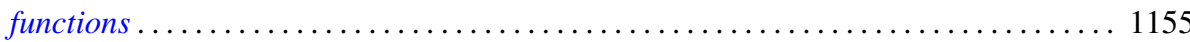

Billy Finney Bryant, On expansive homeomorphisms .................... 1163

Jean W. Butler, On complete and independent sets of operations in finite algebras . . . . . 1169

Lucien Le Cam, An approximation theorem for the Poisson binomial distribution ...... 1181

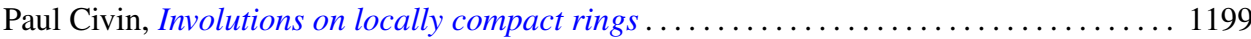

Earl A. Coddington, Normal extensions of formally normal operators . . . . . . . . . 1203

Jacob Feldman, Some classes of equivalent Gaussian processes on an interval ........ 1211

Shaul Foguel, Weak and strong convergence for Markov processes . . . . . . . . . . . 1221

Martin Fox, Some zero sum two-person games with moves in the unit interval ........ 1235

Robert Pertsch Gilbert, Singularities of three-dimensional harmonic functions . . . . . . . 1243

Branko Grünbaum, Partitions of mass-distributions and of convex bodies by

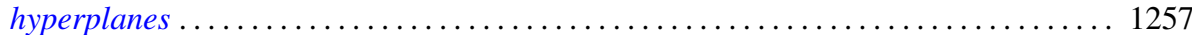

Sidney Morris Harmon, Regular covering surfaces of Riemann surfaces ........... 1263

Edwin Hewitt and Herbert S. Zuckerman, The multiplicative semigroup of integers

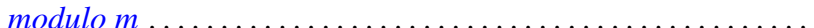

Paul Daniel Hill, Relation of a direct limit group to associated vector groups . ......... 1309

Calvin Virgil Holmes, Commutator groups of monomial groups . .

James Fredrik Jakobsen and W. R. Utz, The non-existence of expansive homeomorphisms

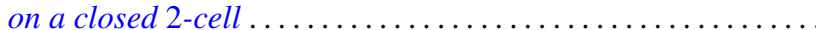

John William Jewett, Multiplication on classes of pseudo-analytic functions . . . . . . . 1323

Helmut Klingen, Analytic automorphisms of bounded symmetric complex domains . . . . 1327

Robert Jacob Koch, Ordered semigroups in partially ordered semigroups . . . . . . . . 1333

Marvin David Marcus and N. A. Khan, On a commutator result of Taussky and

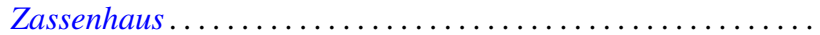

John Glen Marica and Steve Jerome Bryant, Unary algebras......

Edward Peter Merkes and W. T. Scott, On univalence of a continued fraction . . . . . . . 1361

Shu-Teh Chen Moy, Asymptotic properties of derivatives of stationary measures . . . . . 1371

John William Neuberger, Concerning boundary value problems . . . . . . . . . . . 1385

Edward C. Posner, Integral closure of differential rings . . . . . . . . . . . . . . . . . 1393

Marian Reichaw-Reichbach, Some theorems on mappings onto . . . . . . . . . . . . . 1397

Marvin Rosenblum and Harold Widom, Two extremal problems . . . . . . . . . . . . . . . . 1409

Morton Lincoln Slater and Herbert S. Wilf, A class of linear differential-difference

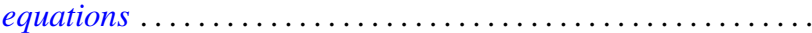

Charles Robson Storey, Jr., The structure of threads . . . . . . . . . . . . . . . . . . 1429

J. François Treves, An estimate for differential polynomials in $\partial / \partial z_{1},, \cdots, \partial / \partial z_{-} n \ldots \ldots 1447$

J. D. Weston, On the representation of operators by convolutions integrals . . . . . . . . 1453

James Victor Whittaker, Normal subgroups of some homeomorphism groups ......... 1469 